\title{
Are Regional Hospital Pharmacies Prepared for Public Health Emergencies?
}

\author{
EDBERT B. HSU, JULIE A. CASANI, AL ROMANOSKY, MICHAEL G. MILLIN, \\ CHRISTA M. SINGLETON, JOHN DONOHUE, E. ROBERT FEROLI, \\ MELVIN RUBIN, ITALO SUBBARAO, DIANNE M. WHYNE, THOMAS D. SNODGRASS, \\ and GABOR D. KELEN
}

Introduction: In the event of a major chemical, biological, radiological, nuclear, or explosive (CBRNE) attack or a natural disaster, large quantities of pharmaceuticals and medical supplies may be required with little or no warning. Pharmaceutical surge capacity for immediate response, before Strategic National Stockpile (SNS) supplies become available, remains a significant gap in emergency preparedness. To date, limited attempts have been made to assess collective regional hospital pharmaceutical response capabilities. In this project, we characterized the level of hospital pharmaceutical response preparedness in a major metropolitan region. Methods: The Johns Hopkins Office of Critical Event Preparedness and Response (CEPAR) convened a collaborative partnership to assess hospital pharmaceutical response capabilities. A survey was developed to characterize pharmaceutical response preparedness to CBRNE threats. Results: All 22 acute care hospitals in the Maryland region were sent pharmaceutical response surveys, and responses were received from $86 \%(19 / 22)$. Within the past year, $84 \%(16 / 19)$ of hospitals had implemented an exercise with pharmacy participation. More than half of the hospitals expect to receive assistance from the SNS in 48 hours or less. Seventy-four percent (14/19) of the hospitals reported an additional dedicated reserve supply for biological events, $74 \%$ (14/19) for chemical events, and $58 \%$ (11/19) for radiological events. Conclusion: Many hospitals in this metropolitan region have taken important steps toward enhancing pharmaceutical preparedness. However, hospitals generally remain underprepared for CBRNE threats and collectively have limited supplies of antibiotics to provide prophylaxis or treatment for hospital staff, their families, and patients in the event of a significant biological incident.

$\mathbf{I}$ THE EVENT of a major attack on the civilian population, large quantities of pharmaceuticals and medical supplies may be required with little or no warning. As government authorities, disaster planners, healthcare pro- fessionals, and public health advocates seek to determine the best ways to mitigate the potential impact of a chemical, biological, radiological, nuclear, or explosive (CBRNE) event or a natural disaster, close coordination

Edbert B. Hsu, MD, MPH; Michael G. Millin, MD, MPH; Italo Subbarao, DO, MBA; Dianne M. Whyne, RN, MS; Thomas D. Snodgrass, BSN; and Gabor D. Kelen, MD, are all with the Office of Critical Event Preparedness and Response and the Department of Emergency Medicine, Johns Hopkins University, Baltimore, Maryland. Julie A. Casani, MD, MPH, and Al Romanosky, $\mathrm{MD}, \mathrm{PhD}$, are with the Office of Emergency Preparedness and Response, and Melvin Rubin, PharmD, is with the Board of Pharmacy, all at the Maryland Department of Health and Mental Hygiene, Baltimore. Christa M. Singleton, MD, MPH, is with the Office of Public Health Preparedness and Response, Baltimore City Health Department. John Donohue, BS, EMT-P, is with Weapons of Mass Destruction and Counterterrorism, Maryland Institute for Emergency Medical Services Systems, Baltimore. E. Robert Feroli, PharmD, is with the Department of Pharmacy, Johns Hopkins Hospital, Baltimore. 
between hospitals and public health systems, as well enhanced partnerships with federal, state, and local agencies, are clearly essential elements of any successful plan.

The Strategic National Stockpile (SNS) was initially developed in 1999 to assist states and communities in responding to public health emergencies. As a repository of antibiotics, antitoxins, life-support medications, and medical/surgical supplies, the SNS is designed to supplement and resupply state and local public health agencies during a declared emergency. The SNS is comprised of "push packages" and vendor managed inventory (VMI) that may be activated in response to different scenarios. Push packages contain a broad spectrum of assets and are strategically positioned for immediate delivery to designated sites within 12 hours of the federal decision to deploy. VMI consists of supplies that may be deployed within 24 to 36 hours for a specific identified threat. ${ }^{1}$

The SNS is an important national resource, although it is not intended for first response but rather as a supplement to state and local responses. Though SNS assets can be deployed rapidly to local and state repositories, logistical challenges of the actual delivery, distribution, and dispensing to people in need may contribute to delays. Furthermore, concerns have been expressed that individual facilities, health departments, and local governments may not be well informed about the processes involved with receipt of SNS supplies. Efforts to strengthen local and state pharmaceutical response preparedness are of highest priority and may have a direct impact on potential lives saved. A relative lack of attention focused on pharmaceutical surge capacity for use during the period immediately following an event, before the SNS may become available, remains a significant gap in emergency preparedness at the local and regional levels.

In many states, each local health department has been assigned the responsibility for developing emergency plans for distributing medications until the SNS resources become available. ${ }^{2}$ In addition, hospitals have been tasked with developing institutional response plans. Although hospitals have taken an accelerated interest in disaster preparedness and are reexamining their disaster plans, competing priorities have left this issue relatively underexamined.

While health departments, working with the federal government, have assumed responsibility for meeting mass prophylaxis needs for the population at large, hospitals are expected to prepare for an immediate and sustained response for up to 72 hours and to be able to meet their own pharmaceutical needs for staff and their families and for patients during public health emergencies. ${ }^{3}$ As demonstrated in the aftermath of Hurricane Katrina, hospitals may be called on to maintain self-sufficiency for extended periods of time. Without proper planning, required supplies may be inadequate, damaged, or ex- pired during an emergency. Furthermore, without predetermined arrangements, local suppliers may be overloaded by simultaneous requests for pharmaceuticals. Anticipated channels for obtaining additional pharmaceutical supplies may prove unreliable due to shortages or the logistical challenges of accessing out-of-state stock. Accordingly, hospital pharmacy staff and administrators must work closely with institutional disaster planners to ensure that critical needs would be adequately met.

To date, limited attempts have been made to assess collective regional hospital pharmaceutical response capabilities. In this project, we characterized the level of hospital pharmaceutical response preparedness in a major metropolitan region.

\section{METHODS}

\section{Development of Partnerships}

In late 2004, the Johns Hopkins Office of Critical Event Preparedness and Response (CEPAR) was charged through the Health Resources and Services Administration (HRSA) Bioterrorism Hospital Preparedness Program (BHPP) in Maryland with assessing current pharmaceutical response capabilities and developing a collaborative pharmaceutical response plan for acute care hospitals in the state. The Pharmaceutical Response Project (PRP) represents a partnership between CEPAR and local and state agencies, including the Maryland Board of Pharmacy, the Maryland Society for Health-System Pharmacists (MSHP), the Department of Health and Mental Hygiene (DHMH), the Baltimore City Health Department (BCHD), the Maryland Institute for Emergency Medical Services Systems (MIEMSS), and the Maryland Emergency Management Agency (MEMA). Designated representatives from each of these agencies were appointed to an advisory panel, and meetings were convened to discuss and address issues related to pharmaceutical availability during public health emergencies. The primary goal of the PRP, as defined by the panelists, was enhancement of regional and statewide pharmaceutical response planning with respect to hospitals in CBRNE incidents, particularly those involving biological agents.

\section{Target Area}

One of the first tasks of the Pharmaceutical Response Project was to define the existing hospital pharmaceutical response capability of a major metropolitan region in the state. Maryland is divided into five Health and Medical Planning Regions. The metropolitan region selected includes Baltimore City and five surrounding counties, 
with an estimated population of 2,466,650. ${ }^{4}$ This region also has approximately half of the state's acute care hospitals (22 of 45).

\section{Survey Development}

A survey was designed to assess current regional pharmaceutical response preparedness and existing capabilities. Key questions were developed to characterize the state of preparedness regarding pharmaceutical response to biological, chemical, or radiological threats and to obtain a detailed assessment of acute care hospitals regarding existing regional pharmaceutical supplies. Other standardized survey instruments, including the CDC Public Health Preparedness and Response Capacity Inventory, ${ }^{5}$ the MIEMSS Maryland Hospital Emergency Preparedness Survey, ${ }^{6}$ and the AHRQ "Understanding Needs for Health System Preparedness and Capacity for Bioterrorist Attacks," 7 were reviewed for items related to pharmaceutical preparedness; these items were incorporated in the PRP survey.

Specifically, the survey elicited information on: (1) regional hospital pharmaceutical response preparedness, including prior establishment of specific protocols and written agreements, access to emergency supply systems, and delineated plans for coordination with SNS assets and exercises conducted; (2) hospital pharmaceutical response capacity for given biological, chemical, and radiological scenarios, including emergency access to supplies; and (3) cataloguing of pharmaceutical supplies by quantity and type.

Additional relevant information, such as hospital characteristics including setting, staffing, and bed capacity, were obtained from other open sources. ${ }^{8}$ The PRP expert panel selected hospital pharmacy directors as the survey respondents, since they were deemed to have greatest knowledge of existing dedicated reserve hospital pharmaceutical supplies. Initial drafts of the survey were developed in collaboration with hospital pharmacists, the Board of Pharmacy, and the MSHP. Draft versions were reviewed and incorporated feedback from each of the partner agencies. The survey was designed to take 25-30 minutes to complete and was successfully piloted with several directors of hospital pharmacies. The survey was then administered to all directors of acute care hospital pharmacies in the study region. The survey can be found at http://www.liebertonline.com/bsp.

\section{RESULTS}

All 22 acute care hospitals in the Maryland region of interest were sent pharmaceutical response surveys. Responses were received from $86 \%$ (19/22) of the acute care hospitals in the region.
The majority of regional hospitals have considered pharmaceutical preparedness as an important component of disaster preparedness planning. Within the past year, $84 \%(16 / 19)$ of hospitals had implemented an exercise with pharmacy participation, including five full-scale drills, eight functional drills, and three tabletop exercises. The responses suggest that hospital pharmaceutical preparedness capabilities are greatest for biological incidents, followed to a lesser degree by preparedness for chemical incidents and radiological incidents. Among the hospitals represented by the survey respondents, 95\% $(18 / 19)$ had assessed their pharmaceutical inventory to determine whether the hospital could support the treatment and prophylaxis of patients exposed to biological agents, 68\% (13/19) had assessed their pharmaceutical inventory for treatment of patients exposed to chemical agents, and 63\% (12/19) had assessed their pharmaceutical inventory for treatment and prophylaxis of patients exposed to radiological agents.

Securing emergency pharmaceutical supply systems is an important aspect of readiness. Fifty-eight percent of the hospitals represented (11/19) had written agreements or memoranda of understanding (MOU) for obtaining or pooling pharmaceutical and medical supplies with other hospitals, offering potential access to supplies from outside institutions. Forty-seven percent of hospitals represented (9/19) had protocols for requesting assistance from the SNS through the local health department, and $68 \%(13 / 19)$ had protocols for securely receiving and transporting emergency pharmaceuticals within the hospital.

Of note, $16 \%$ (3/19) of hospitals expect to receive assistance from the SNS within 12 hours, $16 \%(3 / 19)$ within 24 hours, $21 \%$ (4/19) within 48 hours, and $42 \%$ (8/19) within 72 hours (Figure 1). Overall, more than half of the hospitals expect to receive assistance from the SNS in 48 hours or less based on anticipated delivery times. In the event of their being unable to obtain any outside pharmaceutical supplies, 95\% (18/19) of hospitals believe that their facility would be able to independently maintain patient care standards for 72 hours. When asked whether the hospital, operating at normal capacity, could respond to an aerosolized anthrax incident with an additional 100 patients requiring inpatient treatment, 53\% (10/19) responded that they could operate without outside assistance for at least 72 hours, and an additional $32 \%(6 / 19)$ responded that they could operate without assistance for 48 hours.

The purpose of the survey was to quantify only existing dedicated reserve supplies at each hospital according to strength/concentration, dosage form, and dosage units. A dosage unit was defined as the count of a given specific strength and dosage form. Seventy-four percent $(14 / 19)$ of the hospitals reported an additional dedicated 


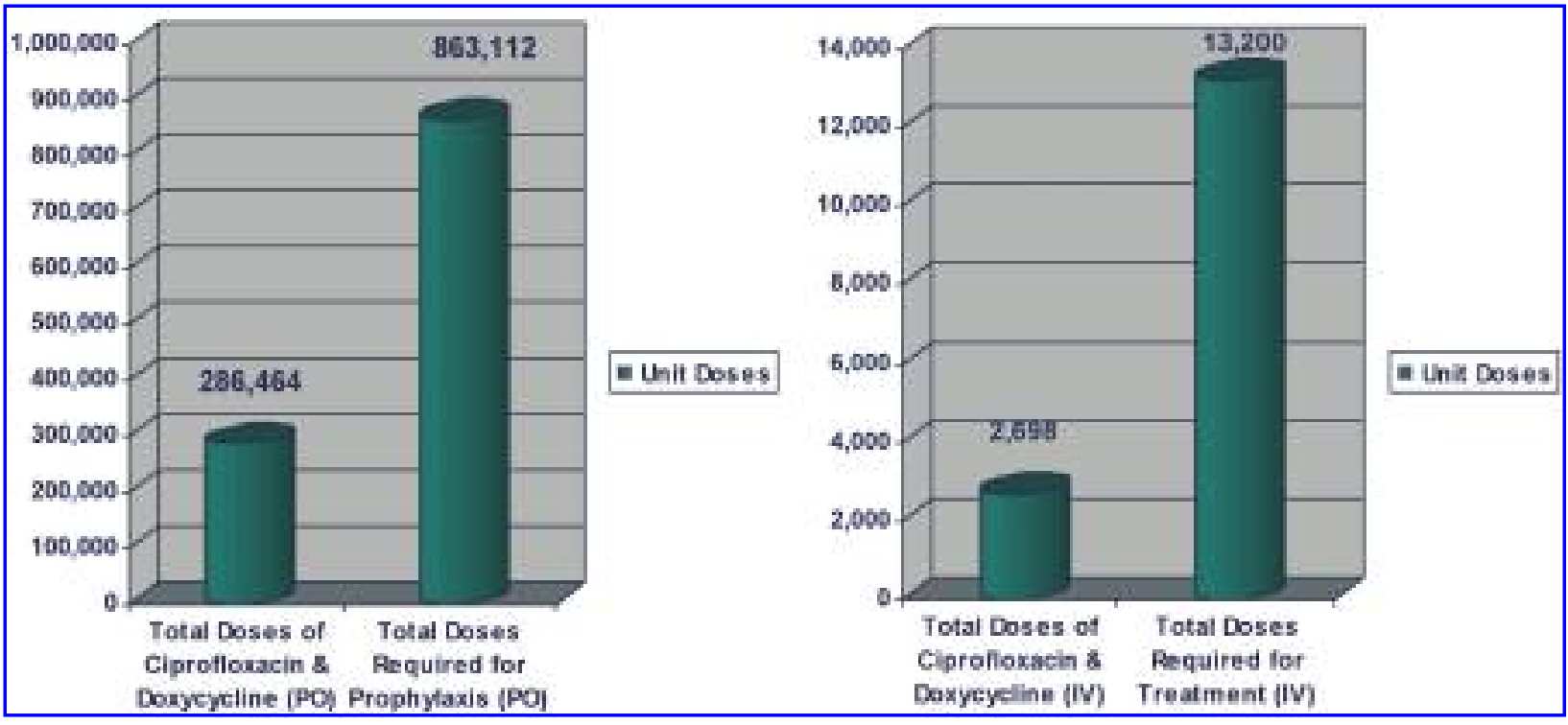

Figure 1. Comparison of Available Doses with Required Unit Doses of Ciprofloxacin and Doxycycline for Prophylaxis And TREATMENT FOR a 72-Hour PERIOD

reserve supply for biological events, 74\% (14/19) for chemical events, and $58 \%(11 / 19)$ for radiological events. On a regional basis, combined hospital doses of ciprofloxacin and doxycycline from the 19 hospitals responding to the survey totaled 286,464 oral doses for prophylaxis and 2,698 parenteral doses for treatment. Included in these totals were 446 regional doses of ciprofloxacin and doxycycline suspension for use in prophylaxis of pediatric populations. A detailed assessment of regional hospital pharmaceutical preparedness is described in Tables 1 and 2.

\section{LIMITATIONS}

One of the limitations of the study is that there was no attempt to characterize all hospital pharmaceutical supplies. Therefore, our results may underestimate the hospitals' collective resources. Hospital pharmaceutical supplies may vary on a daily basis and may already be accounted for during an emergency. Since an existing separate dedicated hospital pharmaceutical supply reflects a more stable measure of hospital preparedness, the survey quantified only these dedicated hospital pharmaceutical caches, rather than all pharmaceutical supplies available for CBRNE incidents in each hospital.

Although respondents were requested to use all sources of information available in completing the survey, survey responses may still not fully and accurately reflect all existing dedicated hospital pharmaceutical reserves. Furthermore, dedicated pharmaceutical reserve supplies represent only one aspect of pharmaceutical re- sponse planning. This project did not address caches that may become available to the hospital or non-hospital populations from public health resources, community health centers, or other government facilities (e.g., local VA hospitals). Hospitals that have fewer pharmaceutical

Table 1. Pharmaceutical Assessment of REgional Hospitals

Hospitals,

Number (\%)

Pharmacy participation in a hospital disaster drill

Written agreement or memorandum of understanding for obtaining pharmaceutical or medical supplies

Protocol for requesting assistance from the SNS

Protocol for how emergency pharmaceuticals could be received and transported

Expect to receive assistance from the SNS in 12 hours

Expect to receive assistance from the SNS in 24 hours

Expect to receive assistance from the SNS in 48 hours

Expect to receive assistance from the SNS in 72 hours

Expect the facility to be able to function independently for up to 72 hours

Expect the hospital to be able to operate at normal capacity with an additional 100 patients for 48 hours

Expect the hospital to be able to operate at normal capacity with an additional 100 patients for 72 hours

$10(53)$ 
Table 2. Pharmaceutical Assessment for Events Involving Biological, Chemical, or Radiological Agents $(N=19)$

\begin{tabular}{|c|c|c|c|}
\hline & $\begin{array}{l}\text { Biological, } \\
\text { Number }(\%)\end{array}$ & $\begin{array}{l}\text { Chemical, } \\
\text { Number }(\%)\end{array}$ & $\begin{array}{l}\text { Radiological, } \\
\text { Number }(\%)\end{array}$ \\
\hline Assessment of pharmaceutical inventory & $18(95)$ & $13(68)$ & $12(63)$ \\
\hline Written plan for prophylaxis & $17(89)$ & NA & $10(53)$ \\
\hline Written plan for treatment & $16(84)$ & $13(68)$ & $11(58)$ \\
\hline Written protocol for dosage requirements & $15(79)$ & $13(68)$ & $12(63)$ \\
\hline $\begin{array}{l}\text { Identified emergency pharmaceutical supply system via local } \\
\text { pharmacies }\end{array}$ & $8(42)$ & $6(32)$ & $5(26)$ \\
\hline $\begin{array}{l}\text { Identified emergency pharmaceutical supply system via } \\
\text { pharmaceutical vendors }\end{array}$ & $6(32)$ & $6(32)$ & $4(21)$ \\
\hline Dedicated reserve supply of pharmaceuticals & $14(74)$ & $14(74)$ & $11(58)$ \\
\hline
\end{tabular}

reserves are not necessarily less prepared; rather, they may have chosen to allocate limited resources in other ways to enhance preparedness (e.g., staff training for biological incidents).

\section{DISCUSSION}

Efforts to describe the regional level of hospital pharmaceutical response preparedness for CBRNE incidents in a major metropolitan area have not been previously reported in the literature. This may reflect the need to balance the sensitive nature of the topic with the importance of bringing increased attention to addressing preparedness needs for CBRNE incidents and other public health emergencies. In exploring hospital pharmaceutical response preparedness, several points are worth noting.

There is a marked disparity between expectations and the likely timeframe for assistance to become available from the SNS. While over half of the hospitals expect to receive assistance from the SNS within 48 hours, the consensus of the PRP panelists was that hospitals must plan to remain self-sufficient and should not expect to receive assistance from the SNS for up to 72 hours (allowing for distribution, delivery, local setup, and dispensing to people in need).

Another striking difference is found between the respondents' perceptions of pharmaceutical capabilities and actual existing reserve pharmaceutical supplies. For example, comparison of reserve doses of parenteral ciprofloxacin and doxycycline at each hospital with the calculated required doses to hypothetically treat an additional 100 patients requiring inpatient treatment for 72 hours demonstrates that only one responding hospital had sufficient reserves to meet these needs.

Considering the current range of pharmaceuticals reported, little uniformity exists in the choice of medications that have been stockpiled. Several hospitals in the region have already developed large stocks of selected pharmaceuticals, most notably ciprofloxacin and doxycycline, while others have limited supplies. Regional pharmaceutical preparedness may be strengthened through more detailed guidance. In support of this, $89 \%$ $(17 / 19)$ of hospitals reported that specific guidelines on maintaining an optimal hospital pharmaceutical cache would be useful in preparedness planning.

Regarding biological threats, recommended prophylaxis and treatment for three of the six CDC Category A biological agents (anthrax, plague, tularemia) include either ciprofloxacin or doxycycline, making these logical choices for stockpiling. ${ }^{9-13}$ Regionally, the combined doses of ciprofloxacin and doxycycline totaled 286,464 oral doses for prophylaxis and 2,698 parenteral doses for treatment (Figure 1). This represents an estimate, as additional smaller quantities of nonstandard dosages and other antibiotics have been identified. The total reserve doses identified fall significantly short of the calculated 863,112 oral doses for prophylaxis of target groups identified by PRP panel consensus (hospital staff, staff families, and inpatients) and the 13,200 parenteral doses for treatment (100 additional patients requiring inpatient treatment for each hospital beyond normal capacity as defined by HRSA) required for a 72-hour period. ${ }^{14}$ The shortfall may reflect lack of clear guidance on how to conduct pharmaceutical preparedness. Other explanations may be that hospitals have not taken into consideration prophylaxis of staff or the family members of staff in their pharmaceutical response planning or that the survey assessment was conducted relatively early in the sequence of phased-in preparedness. Finally, given budget constraints, hospitals simply may not be able to afford these pharmaceuticals.

While there is a regional shortfall of ciprofloxacin and doxycycline needed for prophylaxis and treatment, a number of hospitals in the region have chosen to stock more limited quantities of other medications such as amoxicillin, gatifloxacin, rifampin, clindamycin, and gentamicin. These antimicrobials are effective treatment 
for select biological agents, but they are potentially less cost-effective and would not offer as flexible or efficacious coverage for use during large-scale biological incidents. ${ }^{15-20}$ Furthermore, compliance with emergency use authorization guidelines as well as increased occurrence of adverse side effects with mass prophylaxis must also be considered. All hospitals (19/19) stated that emergency access to a local or regional reserve pharmaceutical stockpile would be useful in preparedness planning. Future research should explore regional stockpile development or augmentation of par levels with rotating stock.

A significant percentage of the population that will require prophylaxis are pediatric patients and those unable to take pills. Planning for pediatric prophylaxis entails considerable cost and effort beyond routine prophylaxis for adult populations. However, provisions for this segment of the population cannot be overlooked. Many children will not be able to take standard medications (i.e., pills) and will require both special formulations and special dosages. Only 446 regional doses of ciprofloxacin and doxycycline suspension were located for use in prophylaxis of pediatric populations. Pharmaceutical response planning for pediatric populations is identified as an important area that has not been adequately addressed.

Specific pharmaceutical preparedness for potential industrial accidents as well as events involving chemical or radiological agents must be given equal consideration. The CHEMPACK Project, a recently developed federal asset deployed in the region, is aimed at threats arising from potential terrorist use of chemical agents. The primary purpose of the CHEMPACK Project is to enhance state and local capabilities and to address sustainability of antidote stocks. With deployment of both EMS and hospital containers, this program has enhanced regional pharmaceutical preparedness for nerve agents. Pharmaceutical preparedness for radiological incidents remains least addressed at the regional level. Limited supplies of potassium iodide/SSKI and chelating agents were found regionally. Cost-benefit analysis of pharmaceutical stockpiling for radiological threats should be explored.

Health-system pharmacists must continue to play a critical role in emergency preparedness efforts and should assume key responsibilities in the planning and execution of pharmaceutical distribution as well as in targeted drug administration to patients in disaster response. According to the American Society of Health-System Pharmacists (ASHP), pharmacists' expertise should be incorporated in: (1) developing guidelines for diagnosis and treatment; (2) selecting appropriate medication use, supply, and distribution for national and regional stockpiles; (3) ensuring proper packaging, storage, handling, labeling, and dispensing of emergency supplies of pharmaceuticals; (4) ensuring appropriate deployment of emergency pharmaceutical supplies; and (5) ensuring ap- propriate education and counseling for individuals who receive pharmaceuticals from an emergency supply in the event of a disaster. ${ }^{21}$

In conclusion, our findings suggest that many hospitals in this metropolitan region have taken important steps toward enhancing pharmaceutical preparedness, yet much remains to be done. Pharmaceutical response planning at the hospital level must be considered in the context of local and national mass prophylaxis strategies and should be designed to support local and regional public health plans. As no single sector is responsible for independent populations, coordination and integration across public and private lines is necessary for global protection. From a pharmaceutical stock standpoint, hospitals in general remain underprepared for CBRNE attacks. Collectively, they have limited supplies of antibiotics to provide prophylaxis or treatment for all hospital staff, their families, and patients in the event of a significant biological incident, and they may not be able to provide surge supplies. However, because of the collaborative efforts of the hospitals, public health departments, and the disaster response community, increased attention to these issues promises to lead to marked improvement in hospital pharmaceutical response preparedness.

\section{ACKNOWLEDGMENTS}

The work of this project was supported through the Health Resources and Services Administration (HRSA) Bioterrorism Hospital Preparedness Program (BHPP) Special Projects Grant in Maryland administered by the Maryland Department of Health and Mental Hygiene (DHMH) and the Maryland Hospital Association (MHA). The authors wish to acknowledge the following individuals for their support of the Pharmaceutical Response Project: Frank Monius (MHA), LaVerne Naesea (Executive Director, Maryland DHMH Board of Pharmacy), Joey Scaletta (DHMH), and Bill Talbott (Maryland Emergency Management Agency).

\section{REFERENCES}

1. U.S. Centers for Disease Control and Prevention. Strategic National Stockpile. Atlanta: U.S. Centers for Disease Control and Prevention; April 14, 2005. Available at: http://www.bt.cdc.gov/stockpile/\#deploy Accessed March 8, 2005.

2. U.S. Code Collection, Title 42, Chapter 6A, Subchapter XXVI, Part B, Number 300hh-12, Strategic National Stockpile. Available at: http://www4.law.cornell.edu/ uscode/htmluscode42 Accessed February 14, 2006.

3. Johns Hopkins Office of Critical Event Preparedness and Response. Pharmaceutical Response Project (PRP) Guid- 
ance Document. Baltimore, Md: Johns Hopkins University \& Johns Hopkins Health System; 2004. Available at: http://www.hopkins-cepar.org/sns/Toolkit/index.html Accessed July 19, 2006.

4. Maryland Department of Health and Mental Hygiene, Office of Public Health Preparedness and Response. Maryland Bioterrorism Hospital Preparedness Program Report. Baltimore: Maryland Department of Health and Mental Hygiene; April 2005. Available at: http://www.cha.state. md.us/ophpr/pdf/April15HRSAReport.pdf Accessed July 17, 2006.

5. U.S. Centers for Disease Control and Prevention. CDC Public Health Preparedness and Response Capacity Inventory. Atlanta: U.S. Centers for Disease Control and Prevention; 2004. Available at: http://www.phppo.cdc.gov/od/ Accessed October 13, 2004.

6. Maryland Department of Health and Mental Hygiene. MIEMSS 2003 Maryland Hospital Emergency Preparedness Survey. Referenced at: http://www.cha.state.md.us/ ophpr/pdf/April15HRSAReport.pdf Accessed July 19, 2006.

7. Agency for Healthcare Research and Quality (AHRQ). Understanding Needs for Health System Preparedness and Capacity for Bioterrorist Attacks. Rockville, Md: Agency for Healthcare Research and Quality; 2002. Available at: http://www.ahrq.gov/about/cpcr/bioterrorism.htm Accessed October 13, 2004.

8. List of Hospitals in Region III of the State of Maryland, September 2004. Available at: http://miemss.umaryland. edu/Chats/Reg3.html Accessed July 19, 2006.

9. U.S. Centers for Disease Control and Prevention. Bioterrorism Agents/Diseases by Category. Atlanta: U.S. Centers for Disease Control and Prevention; undated. Available at: http://www.bt.cdc.gov/agent/agentlist-category.asp Accessed July 17, 2006.

10. PDR Guide to Biological and Chemical Warfare Response: Diagnosis, Treatment, Prevention. Montvale, NJ: Medical Economics Company; 2002.

11. Medical Management of Biological Casualties Handbook. Frederick, Md: U.S. Army Medical Research Institute of Infectious Diseases; 2001.

12. Henderson DA, Ingelsby TV, O'Toole T, eds. Bioterrorism: Guidelines for Medical and Public Health Management. Chicago: AMA Press; 2002.

13. U.S. Centers for Disease Control and Prevention. Fact Sheet: Anthrax Information for Health Care Providers. Atlanta: U.S. Centers for Disease Control and Prevention. Available at: http://www.bt.cdc.gov/agent/anthrax/anthraxhcp-factsheet.asp Accessed July 19, 2006.

14. Health Resources and Services Administration. National Bioterrorism Hospital Preparedness Program: FY2004 Continuation Guidance. Washington, DC: U.S. Department of Health and Human Services; Health Resources and
Services Administration; 2004. Available at: http://www. doh.state.fl.us/demo/php/PDFs/HRSApdfs/hrsa04biot.pdf Accessed July 19, 2006.

15. Update: Investigation of bioterrorism-related anthrax and interim guidelines for exposure management and antimicrobial therapy, October 2001. MMWR Morb Mortal Wkly Rep Oct 26, 2001;50(42):909-919. Available at: http://www.cdc.gov/mmwr/preview/mmwrhtml/mm5042a1. htm Accessed July 18, 2006.

16. U.S. Centers for Disease Control and Prevention. Frequently Asked Questions About Plague. Atlanta: U.S. Centers for Disease Control and Prevention; 2005. Available at: http://www.bt.cdc.gov/agent/plague/faq.asp Accessed July 17, 2006.

17. Inglesby TV, Dennis DT, Henderson DA, et al. Plague as a biological weapon: medical and public health management. Working Group on Civilian Biodefense. JAMA 2000; 283(17):2281-2290.

18. U.S. Centers for Disease Control and Prevention. Key Facts About Tularemia. Atlanta: U.S. Centers for Disease Control and Prevention; 2003. Available at: http://www.bt. cdc.gov/agent/tularemia/facts.asp Accessed July 18, 2006.

19. Dennis DT, Inglesby TV, Henderson DA, et al. Tularemia as a biological weapon: medical and public health management. JAMA 2001;285(21):2763-2773.

20. Markenson D, Redlener I. Pediatric Preparedness for Disasters and Terrorism. A National Consensus Conference. Executive Summary. New York: National Center for Disaster Preparedness, Columbia University Mailman School of Public Health; 2003. Available at: http://www.bt.cdc.gov/ children/pdf/working/execsumm03.pdf Accessed July 18, 2006.

21. American Society of Health-System Pharmacists. ASHP statement on the role of pharmacists in emergency preparedness. In: ASHP Policy Positions, Statements, and Guidelines. Bethesda, Md: American Society of HealthSystem Pharmacists; 2003. Available at: http://www. ashp.org/bestpractices/MedTherapy/Specific_St_EmergPrep.pdf Accessed July 18, 2006.

Manuscript received February 16, 2006;

accepted for publication May 16, 2006.

Address reprint requests to:

Edbert Hsu, MD, MPH

Johns Hopkins University

Office of Critical Event Preparedness and Response 5801 Smith Ave.

Davis Building, Suite 3220

Baltimore, MD 21209

E-mail: edhsu@jhmi.edu 